\title{
Nonimmune hydrops fetalis with intrauterine fetal demise presenting at 41-weeks gestational age: the ultrasonographic findings and case report
}

\author{
Sule MB ${ }^{1^{*}}$, Umar $\mathrm{AU}^{2}$, Gele $\mathrm{IH}^{3}$, Umar $\mathrm{FK}^{3}$, Yunusa $\mathrm{M}^{3}$, Sani $\mathrm{MT}^{3}$, Obi FC ${ }^{3}$ and Olatunji $\mathrm{AK}^{3}$ \\ ${ }^{1}$ Radiology Department, Usmanu Danfodiyo University, Sokoto. \\ ${ }^{2}$ Radiology Department, Gombe State University, Gombe. \\ ${ }^{3}$ Radiology Department, Usmanu Danfodiyo University Teaching Hospital, Sokoto.
}

*Corresponding Author: Sule Muhammad Baba; Department of Radiology, Usmanu Danfodiyo University, Sokoto.

Received date: February 02, 2021: Accepted date: February 26, 2021: Published date: March 11, 2021

Citation: Sule MB, Umar AU, Gele IH, Umar FK, Yunusa M, Sani MT, Obi FC and Olatunji AK (2021 Nonimmune hydrops fetalis with intrauterine fetal demise presenting at 41-weeks gestational age: the ultrasonographic findings and case report. J. of Clin Case Rep and Stu 2(1); DOI: 10.31579/2690-8808/062

Copyright: (C) 2021: Sule Muhammad Baba. This is an open access article distributed under the Creative Commons Attribution License, which permits unrestricted use, distribution, and reproduction in any medium, provided the original work is properly cited.

\begin{abstract}
Hydrops fetalis is the abnormal accumulation of fluid in two or more different fetal compartments and most times associated with polyhydramnios and placental edema, and has an estimated incidence of about 1 in 3000 pregnancies.

A 27-year-old multigravida; G8P7, was referred for the first ultrasonography in the Index pregnancy on account of not feeling/observing fetal movement for about two-weeks. The obstetric ultrasonography demonstrated a single fetus with absent fetal heartbeat, in hyper-flexed position, accumulation of fluid in the abdominal cavity; right sub-diaphragmatic region and in the right pleural cavity in keeping with fetal ascites and fetal effusion. There is overriding of fetal skull with Thickened/edematous placenta with a thickness of about $7 \mathrm{~cm}$, and fetal skin thickness of about $10 \mathrm{~mm}$. There is associated intrafetal gas. The estimated femoral length gave a gestational age approximating 41 -weeks.

A diagnosis of hydrops fetalis most likely non-immune form with fetal demise presenting at 41-weeks gestational age in a multiparous woman with features anemia presumably from sepsis and urinary tract infection (bilateral pyelonephritis and cystitis) in a setting of poor antenatal care was entertained.

The fetus was expelled following induction of labor at the peripheral centre, the patient had transfusion done with commencement of antibiotics for correction of anemia and sepsis with plans of further clinical and laboratory evaluation.
\end{abstract}

We present the ultrasonographic features of hydrops fetalis with intrauterine fetal demise due to its unique presentation.

Keywords: intrafetal gas, fetal death, antenatal care, fluid

\section{Introduction}

Hydrops fetalis (HF) is a term for the abnormal accumulation of fluid in at least two different fetal compartments $[1,2]$. The condition is often characterized by subcutaneous edema, effusions in two or more serous cavities including pericardial or pleural effusions and ascites, with associated polyhydramnios and increased placental thickness [1,3].

Hydrops fetalis is most times regarded as a fatal condition and diagnosed following ultrasonography, and in most cases occurs in rhesus alloimmunization especially before prophylactic anti-D administration in the developed countries, although an entirely different scenario may be encountered in the developing nations [4].

Anti-D immunoglobulin, has reduced the incidence of the immune form of HF, though the non-immune HF has increased in incidence and seen in about $90 \%$ of cases [4-6]. The incidence of HF is between 1 in 1500 and 1 in 3000 pregnancies $[4,7]$.
Hydrops fetalis occurs following an imbalance in the regulation of fluid leading to an increase in interstitial fluid production or a decrease in lymphatic drainage, these leads to accumulation of fluid in fetal soft tissues and serous cavities [8].

Hydrops fetalis has been classified into two forms; the iso-immune HF and the non-immune HF. The immune-HF arises from fetal hemolysis that is caused by circulating maternal antibodies to antigens of fetal red blood cells, the incidence of which has tremendously declined due to the advent of anti-D gamma globulin prophylaxis [9-11].

Etiologically, the nonimmune-HF can occur from varying mechanisms, these may include cardiovascular in $21.7 \%$, idiopathic in $17.8 \%$, genetic in $13.4 \%$, hematological in $10.4 \%$, metabolic in $1.1 \%$ and gastrointestinal causes in about $0.5 \%$, to mention but a few $[2,8,9,12]$.

The mortality rate of non-immune HF is relatively high during fetal or neonatal period making prediction of survival rate challenging, despite improvements in diagnosis and management [9, 13 and 14]. 
Ultrasonography plays a role in detecting the prenatal cause and diagnosis of non-immune HF, though detection of prenatal cause is less common and often requires regular ultrasound follow ups. Etiologies like cardiovascular diseases, anatomic abnormalities and congenital infections are often diagnosed following prenatal and postnatal ultrasonography [1517].

Intrauterine fetal death (IUFD) is defined by the American College of Obstetrician and Gynecologists as death of a fetus past 20 weeks of gestation and or weight of 500grams and above $[18,19]$.

The cause of death of a fetus are basically classified as fetal causes in 25$40 \%$, placental causes in $25-35 \%$, maternal causes in about $5-10 \%$, and idiopathic causes in about 25-35\% [18].

In IUFD, vaginal delivery is preferred except for specific indications, the induction of labor by oxytocin and prostaglandins induction or augmentation given by various routes is applicable [20].

The aim of this study is to present the ultrasonographic features of nonimmune hydrops fetalis and intrauterine fetal demise.

\section{Case Report}

A 27-year-old multigravida, she had seven successful pregnancies and vaginal deliveries; all alive. She is currently pregnant at term coming for the first ultrasonography in the index pregnancy on account of not feeling/observing fetal movement for about two-weeks.

She admitted not to have attended any antenatal visit with the current pregnancy, intake of over-the-counter medications and local herbs and concussions.

No family history of fetal death, sickle cell disease or any congenital malformation/anomaly in the other children and had uneventful prenatal, perinatal and postnatal period for the seven children.
The mother appears not well fed and not ill-looking, not disoriented, not dehydrated, anicteric, acyanosed, not in respiratory distress, no fingerclubbing, and also appeared moderately pale.

The mother had normal blood pressure of $90 / 60 \mathrm{mmHg}$, pulse rate of 86 beats per minute, and respiratory rate of about 15 cycles per minute.

The mother has packed cell volume of about $27 \%$, white blood cell count of 13500 per microlitre and erythrocyte sedimentation rate of about $8 \mathrm{~mm} /$ hour. The blood group is A with a positive rhesus factor; $\mathrm{A}^{+}$.

The mother had an abdominal and pelvic ultrasound scan that demonstrated mild-moderate liver enlargement, with an enlarged span having a length of $165 \mathrm{~mm}$. the kidneys showed increase in cortical reflectivity most likely from pyelonephritis, the urinary bladder showed mobile echoes in urine in keeping with cystitis, no ascites or mass demonstrated, and the remaining abdominal organs appeared normal.

The obstetric ultrasonography demonstrated a single fetus with absent fetal heartbeat and hyper-flexed position, accumulation of fluid in the abdominal cavity; right sub-diaphragmatic region and in the right pleural cavity in keeping with fetal ascites and fetal effusion (figures $1 \& 2$ ). There is overriding of fetal skull with thickened/edematous placental with a thickness of about $7 \mathrm{~cm}$ with skin thickness of about $10 \mathrm{~mm}$ (figures $1 \& 2$ ). The estimated femoral length gave a gestational age approximating 41weeks.

A diagnosis of hydrops fetalis most likely non-immune form with fetal demise presenting at 41-weeks gestational age in a multiparous woman with features anemia presumably from sepsis and urinary tract infection (bilateral pyelonephritis and cystitis) in a setting of poor antenatal care was entertained.

The fetus was expelled following induction of labor at the peripheral centre, the patient had transfusion done with commencement of antibiotics for correction of anemia and sepsis with plans of further clinical and laboratory evaluation.

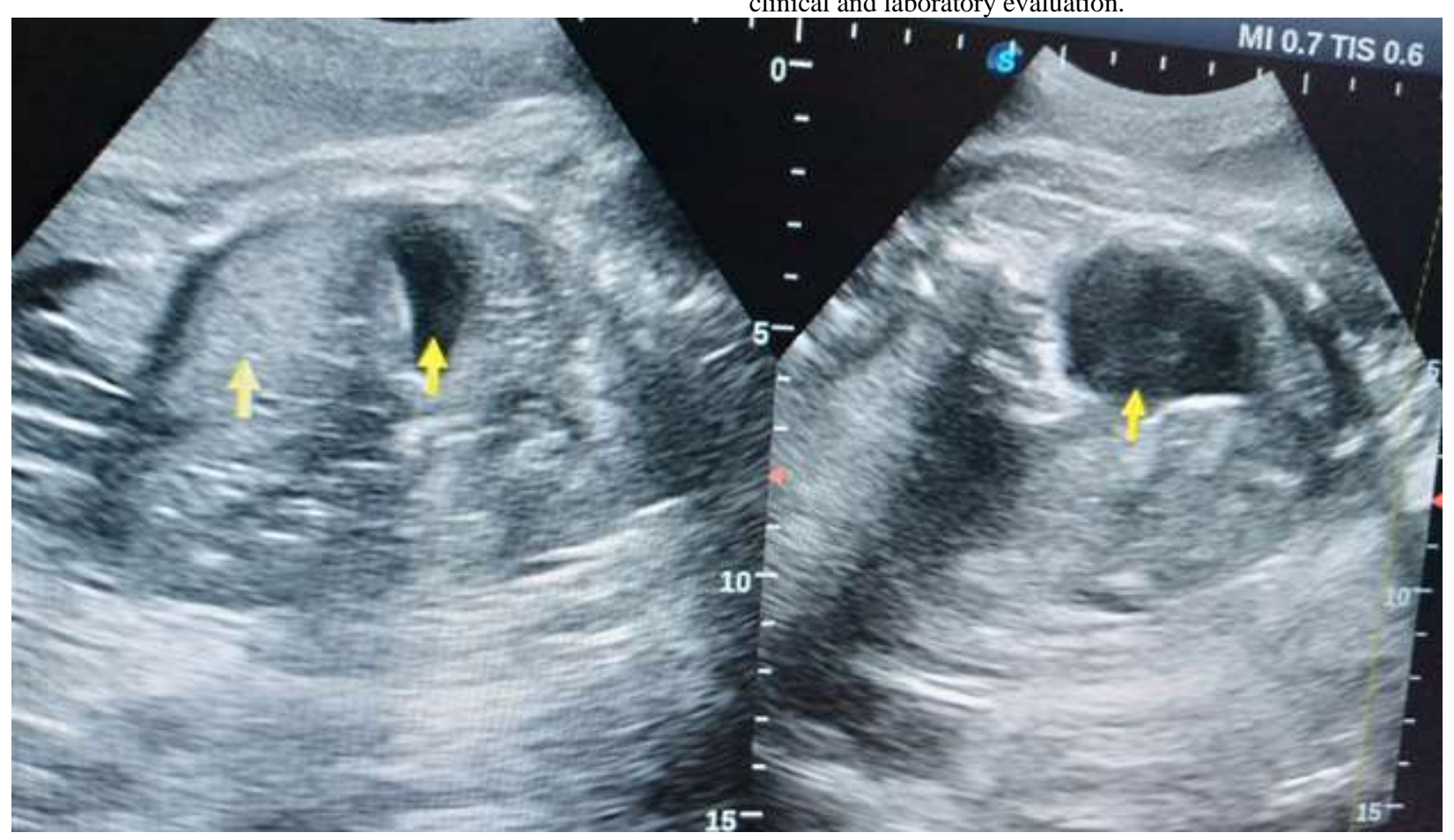

Figure 1: Obstetric ultrasonogram, demonstrating the liver and sub diaphragmatic fluid collection on the left image, these are been indicated by yellow arrows respectively, the thickened placenta and fetal skin thickness also demonstrated on the left image. A view of the pocket of fetal ascites is clearly demonstrated on the right image. 


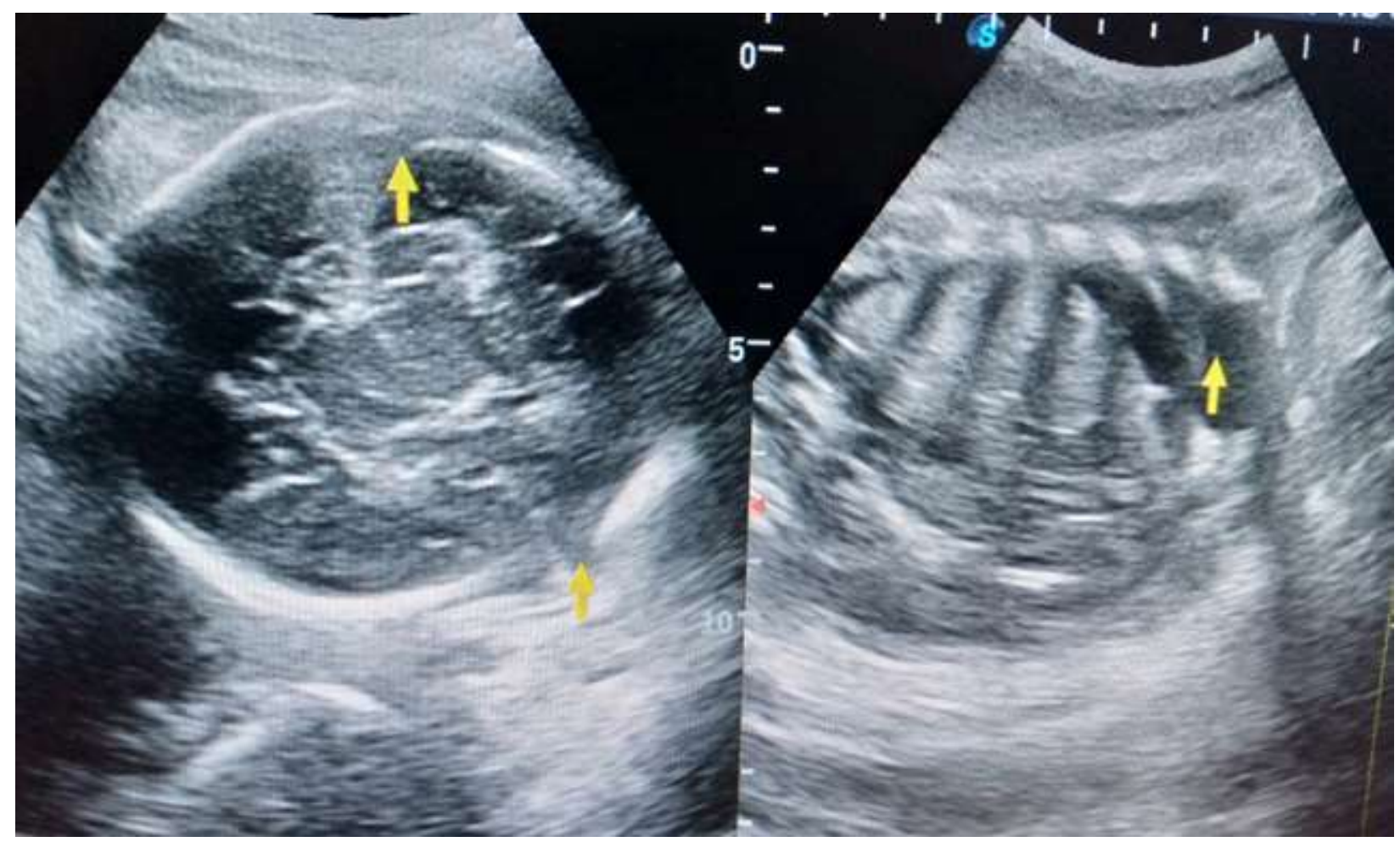

Figure 2: An obstetric ultrasonogram showing overriding of the skull bones of about $22 \mathrm{~mm}$ on the left image, the right image demonstrates fluid within the pleural space, note the overlying ribs.

\section{Discussion}

Hydrops fetalis is the abnormal fluid collection in two or more fetal compartments such as skin, ascites, pleural and pericardial effusion [1-3]. The index case had accumulation of fluid in the pleural and peritoneal spaces with the overlying skin, thereby conforming to what is reported in the literatures.

The condition (HF) is often characterized by subcutaneous edema, effusions in two or more serous cavities including pericardial or pleural effusions and ascites, with associated polyhydramnios and increased placental thickness $[1,3]$. The index case had subcutaneous edema with a skin thickness of about $10 \mathrm{~mm}$, had fluid collection in the abdominal and pleural cavities, the placental thickness was about $7 \mathrm{~cm}$, conforming to these literatures.

Hydrops fetalis has been classified into two forms; the iso-immune HF and the non-immune HF. The immune-HF arises from fetal hemolysis that is caused by circulating maternal antibodies to antigens of fetal red blood cells, the incidence of which has tremendously declined due to the advent of anti-D gamma globulin prophylaxis [9-11]. The mother of the index case never had administration of anti-D gamma globulin prophylaxis ruling out the possibility of the immune-HF, thereby conforming to these literatures.

Non-immune HF is a term that defines an edematous fetus that does not have erythroblastosis fetalis from isoimmunization [21]. The index case is most likely the non-immune type, no history or features to suggest isoimmunization was gotten from the mother, she happens to be of the Rhesus positive group, thereby conforming to this literature.

The mortality rate of non-immune HF is relatively high during fetal or neonatal period making prediction of survival rate challenging, despite improvements in diagnosis and management [9, 13 and 14]. The index case was diagnosed in the prenatal/fetal stage and presented as a case of fetal demise, conforming to these literatures.
Etiologically, the nonimmune-HF can occur from varying mechanisms, these may include cardiovascular in $21.7 \%$, idiopathic in $17.8 \%$, genetic in $13.4 \%$, hematological in $10.4 \%$, metabolic in $1.1 \%$ and gastrointestinal causes in about $0.5 \%$, to mention but a few $[2,8,9,12]$. The case under review is most likely from the idiopathic cause, however maternal sepsis and urinary tract infections are been entertained, thereby conforming to these literatures.

Hydrops fetalis been a serious fetal condition, is usually diagnosed following ultrasonography, this can also detect some of the possible causes, the index case was also diagnosed following ultrasound examination, thereby conforming to these literatures.

The death of a fetus that occurs after the $20^{\text {th }}$ week of intrauterine life is regarded as intrauterine fetal death/demise, prior to which is termed a miscarriage. About $1 \%$ of normal uncomplicated pregnancies end in fetal death while $15 \%$ of IUFD has no identified cause [22].

The ultrasound features of IUFD are absent fetal heartbeat, absent fetal movements, overlapping skull bones (Spalding sign), soft tissue edema (skin thickness greater than $5 \mathrm{~mm}$ ), distorted fetal anatomy, intrafetal gas and echogenic amniotic fluid [22]. The index case has almost all of these features, thereby conforming to this literature.

The incidence of intrauterine fetal death is higher in the developing nations and often associated with maternal anemia, pregnancy-induced hypertension, illiteracy, congenital malformations and low socioeconomic status [23], the index case had association of most of these conditions, thereby conforming to this literature.

The treatment of HF is directed at the cause, the index case was diagnosed as a case of fetal demise, the fetus was expelled from induction of labor as reported by Bhatia, et al [20].

\section{Conclusion}


Good antenatal care with adequate laboratory and imaging evaluation are vital in detecting cases of hydrops fetalis, so as to institute adequate and appropriate management to reduce antecedent complication and fetal demise.

\section{References}

1. Kumar M, Jha V, Singh A. (2018) Nonimmune Hydrops Fetalis: Factors Which Predict Outcome. J Obstet Gynaecol India; 68: 197-203.

2. Bellini C, Hennekam RC. (2012) Non-immune hydrops fetalis: a short review of etiology and pathophysiology. Am J Med Genet A.; 58A: 597-605.

3. Randenberg AL. (2010) Nonimmune hydrops fetalis part I: etiology and pathophysiology. Neonatal Netw; 29: 281-295.

4. Sekar R. Hydrops Fetalis. IntechOpen. 2019:1-13.

5. Bellini C, Hennekam RC, Fulcheri E. (2009) Etiology of nonimmune hydrops fetalis: A systemic review. Am J Med Genet Part A; 149A: 844-851.

6. Steurer MA, Peyvandi S, Baer RJ. (2017) Epidemiology of live born infants with nonimmune hydrops fetalis-insights from a population-based dataset. J Pediatr; 1:182-188.

7. Hutchinson AA, Drew JH, Yu VY. (1982) Nonimmunologic hydrops fetalis: A review of 61 cases. Obstet Gynecol; 59:347352.

8. Kosinki P, Krajewski P, Wielgos M, Jezela-Stanek A. (2020) Nonimmune Hydrops Fetalis-Prenatal Diagnosis, Genetic Investigation, Outcomes and Literature Review. J Clin Med. 9:1789.

9. Meng D, Li Q, Hu X, Wang L, Tan S, Su J, et al. (2019) Etiology and Outcome of non-immune Hydrops Fetalis in Southern China: report of 1004 cases. SCIENTIFIC REPORTS. 9:10726.

10. Heinonen S, Ryynanen M, Kirkinen P. (2000) Etiology and outcome of second trimester non-immunologic fetal hydrops. Acta obstetrician et gynecologica Scandinavica. 79:5-8.

11. Machin GA. (1989) Hydrops revisited: literature review of 1,414 cases published in the 1980s. Am J Med Genet. 34:366-390.
12. Bellini C, Donarini G, Paladini D, Calevo MG, Bellini T, Ramenghi LA, et al. (2015) Etiology of non-immune hydrops fetalis: An update. Am J Med Genet. Part A. 167:1082-1088.

13. Yeom W, et al. (2015) Clinical characteristics and perinatal outcome of fetal hydrops. Obstet Gynecol Sci. 58:90-97.

14. Fukushima K et al. (2011) Short-term and long-term outcomes of 214 cases of non-immune hydrops fetalis. Early human development. 2011; 87:571-575.

15. Swearingen C, Colvin ZA, Leuthner SR. (2020) Non-immune Hydrops fetalis. Clin Perinatol. 47:105-121.

16. Gonce A, Marcos MA, Borrell A, Lopez M, Nadal A, Figueras F, et al. (2012) Maternal IgM antibody status in confirmed fetal cytomegalovirus infection detected by sonographic signs. Prenat Diagn. 2012; 32:1-5.

17. Syridou G, Spanakis N, Konstantinidou A, Piperaki ET, Kafetzis D, Patsouris E, et al. (2008) Detection of cytomegalovirus, parvovirus, B19 and herpes simplex viruses in cases of intrauterine fetal death: Associaion with pathological findings. $J$ Med Virol. 80:1776-1782.

18. Saha D, Kurude VN, Mundhe S. (2019) A study of intrauterine fetal death in a tertiary care hospital. Int J Reprod Contracept Gynecol. 8:2647-2651.

19. American College of Obstetricians and Gynecologists. Diagnosis and management of fetal death. ACOG Technical Bulletin no 176. Int J Gynecol Obstet. (1993) 42:291-299.

20. Bhatia Tulsi, Narshetty JG, Bagade P, Amit Kulkarni, Rai M. (2016) Clinical study of cases of intrauterine fetal death in a tertiary centre. Int J Res Med Sci. 4:800-805.

21. Burke SME. (1999) Nonimmune Hydrops Fetalis. J Perinat Neonat Nurs. 13:33-44.

22. Sharma S, Sidhu H, Kaur S. (2016) Analytical study of intrauterine fetal death cases and associated maternal conditions. J Appl Basic Med Res. 6:11-13.

23. Bell DJ, Refaey M. (2021) Fetal death in utero.radiopedia.org. 